\title{
Selection, Mutations and Codon Usage in Bacterial Model
}

\author{
Franco Bagnoli* \\ Dipartimento di Matematica Applicata, \\ Università di Firenze, \\ via S. Marta 3, \\ I-50139 Firenze, Italy. \\ Pietro Liò \\ Dipartimento di Biologia Animale e Genetica, \\ Università di Firenze, \\ Via Romana 17, \\ I-50125 Firenze, Italy
}

\begin{abstract}
We present a statistical model of bacterial evolution based on the coupling between codon usage and tRNA abundance. Such a model interprets this aspect of the evolutionary process as a balance between the codon homogenization effect due to mutation process and the improvement of the translation phase due to natural selection. We develop a thermodynamical description of the asymptotic state of the model. The analysis of naturally occurring sequences shows that the effect of natural selection on codon bias not only affects genes whose products are largely required at maximal growth rate conditions but also gene products that undergo rapid transient increases.
\end{abstract}

appeared as J. theor. Biol. 173, 217 (1995).

\section{INTRODUCTION}

The evolution of the genetic material is determined by the interactions among mutations, random drift and natural selection.

The mutation rate seems to vary both among and within genomes, being affected by many factors, such as the chromosomal position (Sharp et al., 1989), the $\mathrm{G}+\mathrm{C}$ content (Wolfe, 1991), the nearest neighbor bases (Blake et al., 1992), the different efficiency of the repair systems between the lagging and the leading DNA strands during replication and

\footnotetext{
*also INFN and INFM, sezione di Firenze, Largo E. Fermi, 2 I-50125 Firenze, Italy
} 
transcription (Veaute \& Fuchs, 1993). Thus the molecular clock seems to tick at different rate for different DNA positions.

The natural selection acts as a driving force at virtually all levels of the genetic information processing and biological organization: from the DNA stability, replication and transcription to messenger RNA life span and translation efficiency, to the correct functioning of the gene products in the building up and propagation of a living organism. Although in principle all these constraints could interact in a very complex way, it is indeed fruitful to try to untangle the role of each element.

We have focused on the translation process as a major source of fitness for the bacterial cell.

Because of the degeneracy of the genetic code and the historical process of codon capture (Osawa \& Jukes, 1989), different codons (synonymous codons) specify for the same amino acid. The bias in the codon usage is generally not random and it is very marked in some procaryotic and eucaryotic genes. The codon choice is specie-specific (Grantham et al., 1980), being roughly the same, although with different intensities, in all the genes within a genome. The codon bias is thought to be an effect of the improvement of the efficiency of the translation process under natural selection.

Several authors have stressed that synonymous codons in E. coli, preferentially used in highly expressed genes, are translated by the most abundant iso-acceptors tRNA species and that the levels of tRNAs in bacterial cells depend on the amino acid usage in proteins (Ikemura, 1981a).

A good correlation between codon usage and tRNAs population has also been found in Mycoplasma capricolum (Yamao et al., 1991), in yeast (Ikemura, 1981b) (Ikemura, 1985) and in chloroplast genomes (Morton, 1993).

The importance of codon usage as a substrate for natural selection is well proved by the coding strategy of the coli phage T4: the early expressed genes of $T 4$ have a codon pattern close to that of $E$. coli while the late expressed genes show a preference towards the codons read by the tRNAs expressed by the genome of the phage itself (Cowe \& Sharp, 1991).

The codon usage influences the elongation rate in the translation process, being the pairing between codon and anticodon of the diffusing tRNA the rate-limiting step compared with the peptide bond formation and translocation steps (Gouy \& Grantham, 1980), but it cannot be directly related to the protein expression rate. In fact many experiments have demonstrated that other crucial steps affecting the protein production rate are the selection of mRNA by a ribosome, the initiation phase and the phenomenon of polypeptidyl-tRNA drop off (Menninger, 1978).

Moreover the dynamic of elongation phase seems to be more complex, depending also by the differences in the energy of codon anticodon pairing (Grosjean \& Fiers, 1982) (Thomas et al., 1988); thus differences in elongation rates have been found for codons read by the same tRNA species (Sorensen \& Pedersen, 1991).

Different considerations should be drawn on the evolutionary role of either the minor or the major codons. Some authors have suggested that selection of some of the rarer tRNA species may be the rate-limiting step in protein synthesis (Varenne et al., 1984): they hypothesize that the rate of polypeptide elongation could act as a regulatory mechanism in gene expression when clusters of rare codons undergo translation, i.e. when the required tRNA concentration is low and rate-limiting. This hypothesis has been confirmed exper- 
imentally by manipulating the concentrations of some tRNAs and creating rate-limiting conditions. Varenne (Goldman, 1982) (Varenne et al., 1984) demonstrated experimentally that the presence of rare codons in highly expressed coding regions is associated with pauses in the synthesis of proteins. Many different contexts have been tested with similar results: Hoekema (Hoekema et al., 1987) replaced some major codons present in the first region of the PGK1 gene of Saccharomices cerevisiae with synonymous minor codons; Kinnaird (Kinnaird et al., 1991) generated a cluster of three rare codons in the GDH gene in Neurospora crassa. Chen and Inouye (Chen \& Inouye, 1990) have demonstrated that the introduction of a cluster of a rare codon, $A G G$, in lacZ sequence of $E$. coli, lowers the expression of the gene; moreover this effect is inversely correlated with the distance between the initiation site and the position of rare codons.

Models that takes into account the influence of minor codons in the initiation region of mRNA for protein production rate have been presented (Liljenstrom \& von Heijne, 1987) (Liljenstrom \& Bomberg, 1987).

On the contrary Sharp and Li (Sharp \& Li, 1986) have hypothesized that the pattern of synonymous codon usage in regulatory genes reflects primarily the relaxation of natural selection. In fact, the absence of rare codons from highly expressed genes may well result from negative selection, while in low expressed genes, selection against rare codons is very weak and so they can accumulate under the pressure of mutation.

Remarkably different considerations should be drawn for the major codons, i.e. codons read by the most abundant tRNAs. There is evidence that the major proteins are translated faster than other proteins and that the elongation rate at major codons is faster than that at other codons (Emilsson \& Kurland, 1990a).

Some authors (Emilsson \& Kurland, 1990a) (Emilsson \& Kurland, 1990b) have hypothesized that growth rate, in bacteria, depends on tRNA abundance. Such relation results in the so called growth maximization strategy and it is consistent with a major codon preference strategy, in which an optimal subset of codons is thought to be used very frequently in highly expressed genes, and their cognate tRNA species are supposed to increase considerably at high growth rates conditions. This is in agreement with the fact that the genes for the major tRNA species are located inside rRNA operons for a coordinate expression.

The growth rate dependence on tRNA abundances and other aspects of the evolution of genes and genomes in bacteria can be faced with statistical mechanics tools. Let us consider an homogeneous population of bacterial genomes, subjected only to synonymous mutations and natural selection. If we study its evolution in the proximity of a steady dynamical state, as for instance a balanced growth state, we can interpret the interaction between mutations and natural selection as a competition between randomness and order. In this spirit we develop a statistical model from which bacterial evolution emerges as an optimization process under environmental constraints.

\section{THE MODEL}

In order to study quantitatively the competition between mutation and natural selection in naturally occurring sequences, we concentrate on the process of the elongation phase. We suppose that the rate limiting step in this phase is the relative abundance of charged 
tRNAs. Thus we assume that the time required by a ribosome to process a codon is inversely proportional to the abundance of the charged cognate tRNAs in its vicinity. Taking into account the data from Gouy and Grantham (Gouy \& Grantham, 1980), the process of tRNA diffusion in cell extract results much faster than all others processes involved in our schematization, and thus we do not consider the effects of spatial gradients.

Furthermore, we assume that the efficiency of aminoacyl-tRNA synthetases is not rate limiting, i.e. that the concentration of the charged tRNAs does not vary with the translation process regardless of the codon composition of mRNAs. This approximation is not completely fulfilled in real bacteria. From the same source above (Gouy \& Grantham, 1980) we obtain that in average only $85 \%$ of the tRNA pool is acylated, indicating that the reaction rate of synthetase is not substrate limited.

In this approximation we do not take into account the finite size of a ribosome, nor the queuing of ribosomes, thus disregarding the positional effects of codon usage, as considered elsewhere (Liljenstrom \& Bomberg, 1987).

With this assumption, the translation time does not depend on the order of codons in mRNA and can be calculated as the sum of the time required for each codon. Finally, we do not consider the influence of fluctuations, i.e. we develop a sort of "mean field" model for the translation.

We indicate the portion of mRNA that code for a protein (or, alternatively, the whole mRNA) with a vector $\mathbf{c} ; c_{i}(i=1, \ldots, 61)$ being the number of codons of type $i$ and $L=\sum_{i} c_{i}$ the length of the coding region. We denote with $a_{k}$ the relative abundance of acylated tRNA of type $k\left(k=1, \ldots, n_{t R N A} ; \sum_{k=1}^{n_{t R N A}} a_{k}=1\right)$. For $E$. coli $n_{t R N A}=45$, see also the following section. The "translational efficiency" of codons by tRNAs is represented by means of a matrix $R_{i k}$ of size $61 \times n_{t R N A}$. Again from (Gouy \& Grantham, 1980), the transpeptidation and translocation phases do not weight heavily on the tRNA cycle, and disregarding them, the mean time required to translate the codon $i$ is inversely proportional to $\rho_{i}=\sum_{k=1}^{n_{t R N A}} R_{i k} a_{k}$. The mean translation time per codon $\tau$ (in arbitrary time units) is thus given by

$$
\tau(\mathbf{c})=\frac{1}{L} \sum_{i=1}^{61} \frac{c_{i}}{\rho_{i}} .
$$

For simplicity, the elements $R_{i k}$ can be taken to be either 0 , for a tRNA $k$ that does not pair with a codon $i$, or 1 , disregarding the differences in energies in the codon-anticodon pairing discussed in the previous section.

In bacteria, cell division and DNA replication are coupled with cell growth (Zyskind \& Smith, 1992) (Ageno, 1992). In balanced growth conditions the average number of genomes, the average number of cells and the cell mass increase exponentially with the same rate.

The cell growth largely depends on the production rate of the most abundant biopolymers, among which ribosomal proteins constitute the larger fraction. The time $\tau$ in formula (1), calculated for an "average" ribosomal protein, can be considered proportional to the mean duplication time. We assume that the natural selection tends to lower this time. A somewhat opposite effect is due to the mutation process, that tends to randomize the codon sequence. Since silent mutations do not change the coded aminoacid and thus they do not 
affect the composition of proteins, they are neutral for the selection over the protein functionality. However, silent mutation are not neutral for the calculation of the duplication time $\tau$, because of the presence of the abundance of cognate tRNAs in formula (酉).

Let us start from a simplified situation, in which two synonymous codons (say codon 0 and 1) are only read by two tRNAs (with respectively abundances $a_{0}$ and $a_{1}$ ), while all other codons are grouped together (say codon 2) and in average are read by tRNAs with abundance $a_{2}\left(a_{0}+a_{1}+a_{2}=1\right)$. The number of synonymous codons is $c_{0}+c_{1}=l$. We consider only mutations between 0 and 1 and vice versa. Since $\tau$ does not depend on the order of the codons in the string, the various strains can be grouped together according with the number $j$ of one's in the mRNA $\left(j=c_{1}\right)$. For each group $j$ there are $g_{j}=\left(\begin{array}{l}l \\ j\end{array}\right)$ strains.

We rewrite the formula (11) for the time $\tau_{j}$ required to duplicate a bacterium belonging to group $j$ as

$$
\begin{aligned}
\tau_{j} & =\left(\frac{j}{a_{1}}+\frac{l-j}{a_{0}}+\frac{L-l}{a_{2}}\right)=r j+q+z ; \\
r & =\frac{a_{0}-a_{1}}{a_{0} a_{1}} ; \quad q=\frac{l}{a_{0}} ; \quad z=\frac{K l}{a_{2}} ; \quad K=\frac{L-l}{l} .
\end{aligned}
$$

The quantity $K^{-1}$ is the relative abundance of the synonymous codons 0 and 1 in the coding region. For $l \ll L, K \rightarrow \infty$; for $l \simeq L, K \simeq 0$. Since selection only acts on 0 and 1 codons, $K^{-1}$ give an indication of the influence of selection on the gene.

In the unit time interval, the number of duplications $\nu_{j}$ of the mass $M_{j}$ of bacteria belonging to group $j$ is $\nu_{j}=\tau_{j}^{-1}$, and thus

$$
\frac{d M_{j}}{d t}=\nu_{j} M_{j}
$$

where for simplicity we set all constants to 1 and we neglect the time dependence of $M_{j}$.

In the limit of very low mutation rate, we can assume that for each generation there is at most only one synonymous mutation, that changes codon 1 to 0 or vice versa. Let us indicate with $\mu / l$ the rate of mutation per codon $(\mu \leq 1)$. The average fraction of bacteria in group $j$ that undergo a mutation per unit of time is $\mu \nu_{j}$. For bacteria in group $j$, a synonymous mutation changes $j$ of one unit. Including mutations, and working with the mass $m_{j}=M_{j} / g_{j}$ of a single strain in group $j$, formula (3) becomes

$$
\frac{d m_{j}}{d t}=(1-\mu) \nu_{j} m_{j}+\frac{j}{l} \mu \nu_{j-1} m_{j-1}+\frac{l-j}{l} \mu \nu_{j+1} m_{j+1}
$$

for $0 \leq j \leq l$, assuming that $\nu_{-1}=\nu_{l+1}=0$.

The total mass of the bacterial population is $M=\sum_{k=0}^{l} g_{k} m_{k}$. We can derive from eq. (田) the evolution equation for the distribution probability $p_{j}=m_{j} / M$ of different strains in the total population. In a natural environment the exponential growth periods are sporadic, generally followed by starvation phases. We mimic this alternation by means of the normalization of distribution, assuming that the strains corresponding to a very low probability are those eliminated by natural selection. 
Since mutations do not change the total mass of population, we have, summing up over $j$ in eq. (四)

$$
\frac{d M}{d t}=\sum_{k=0}^{l} g_{k} \nu_{k} m_{k}=M \sum_{k=0}^{l} g_{k} \nu_{k} p_{k}=M \bar{\nu}
$$

obtaining

$$
\frac{d p_{j}}{d t}=\frac{d}{d t} \frac{m_{j}}{M}=\frac{1}{M} \frac{d m_{j}}{d t}-\frac{m_{j}}{M^{2}} \frac{d M}{d t}
$$

and thus

$$
\begin{aligned}
\frac{d p_{j}}{d t} & =\left[(1-\mu) \nu_{j}-\bar{\nu}\right] p_{j}+\frac{j}{l} \mu \nu_{j-1} p_{j-1}+\frac{l-j}{l} \mu \nu_{j+1} p_{j+1} \\
\bar{\nu} & =\sum_{k=0}^{l} g_{k} \nu_{k} p_{k} .
\end{aligned}
$$

Before dealing with these equations from a mathematical point of view, let us put some thermodynamical considerations. The scenario is reminiscent of statistical mechanics systems, in which there is competition between order (the energy function to be minimized, related to the average duplication time) and the entropy, mutuated by the temperature.

Each strain in group $j$ contributes with $\tau_{j}$ to the mean duplication time. Taking into account the analogy between the duplication time of strains and the energy levels of a statistical system, in the equilibrium (stationary) state we can tentatively apply the methods from equilibrium statistical mechanics. The maximization of the "entropy" $S=-\sum_{k=0}^{l} g_{k} p_{k} \ln p_{k}$ under the constraints $\sum_{k=0}^{l} g_{k} p_{k}=1$ (normalization of probability distribution) and $\sum_{k=0}^{l} g_{k} p_{k} \tau_{k}=$ const (in order to select the most probable probability distribution within those with the same fitness), gives the Boltzmann distribution (Landau \& Lifshitz, 1958)

$$
p_{j}=C \exp \left(-\beta \tau_{j}\right) .
$$

The Lagrange multiplier $\beta$ can be considered as the inverse of an effective "temperature" $T$, that, intuitively, should be related to the mutation rate $\mu$. In the stationary state, $\bar{\nu}$ is constant in time, and from eq. (5), $M$ (and thus $m_{j}$ ) grows exponentially.

Inserting the Ansatz

$$
m_{j}=M_{0} \exp \left(\alpha t-\beta \tau_{j}\right)
$$

( $M_{0}$ is the total mass at time $t=0$ ) in the equation (4), and using the relations (2), we get for the asymptotic state

$$
(1-\mu) \nu_{j}-\alpha+\frac{l-j}{l} \mu \nu_{j+1} x+\frac{j}{l} \mu \nu_{j-1} \frac{1}{x}=0,
$$

where $x=\exp (-\beta r)$. Approximating $\nu_{j+1} \simeq \nu_{j-1} \simeq \nu_{j}$, with an error of order $r / \tau_{j}^{2}$, which is small for $l$ or $L$ large or $a_{1}-a_{0}$ small, eq. (7) becomes a linear equation in $j$. This equation holds independently of $j$ if

$$
\begin{aligned}
& x=\frac{-(1-\mu) \operatorname{lr}+\sqrt{(1-\mu)^{2} l^{2} r^{2}+4 \mu^{2} q(q+\operatorname{lr})}}{2 \mu(q+\operatorname{lr})}, \\
& \alpha=\frac{\mu\left(1-x^{2}\right)}{\operatorname{lr} x} .
\end{aligned}
$$


Using the relations (21) we get

$$
x=\frac{(1-\mu)\left(a_{1}-a_{0}\right)+\sqrt{(1-\mu)^{2}\left(a_{1}-a_{0}\right)^{2}+4 \mu^{2} a_{0} a_{1}\left(1+K \tilde{a}_{0}\right)\left(1+K \tilde{a}_{1}\right)}}{2 \mu a_{0}\left(1+K \tilde{a}_{1}\right)}
$$

where $\tilde{a}_{i}=a_{i} / a_{2}, i=0,1$.

Note that $\alpha=\bar{\nu}$. Numerical simulations of eq.(16) agree very well with this solution, and show that it is the only stable solution for almost all initial distributions.

The asymptotic form of $M_{j}=g_{j} m_{j}$ for $K=0, l \rightarrow \infty$ and $1 \ll j \ll l$ is

$$
M_{j}=M_{0} \frac{2^{l} \sqrt{2}}{\sqrt{\pi l}} \exp \left(\alpha t-\frac{(2 j-l)^{2}}{2 l}-\beta \tau_{j}\right) .
$$

It is remarkable that although the distribution of $p_{j}$ is always a growing function of $j$ (for $a_{1}>a_{0}$ ), the distribution $M_{j}$ is bell shaped, due to the contribution of the multiplicity factor $g_{j}$.

The most probable group $j_{\max }$ corresponding to the maximum of $M_{j}$ is, using the Stirling approximation for $g_{j}$,

$$
j_{\max }=\frac{l x}{x+1} .
$$

For $L \gg l(K \rightarrow \infty), x=1, T \rightarrow \infty, \alpha=0$ and $j_{\max }=l / 2$. The distribution $p_{j}$ is flat because in this case the selection has no effect. This is due to the effective neutrality of mutations inside a group $j$.

Let us consider in the following the opposite case $L=l(K=0)$, that represents a bacterium in which an essential protein is totally composed by the single aminoacid coded by 0 and 1 codons. This rather unrealistic case makes our model equivalent to a $1 \mathrm{D}$ kinetic Ising model (Kawasaki, 1972) in an external field without interactions among spins. In this case we can analize in more details the influence of selection.

We can express $\alpha$ and $\beta$ in term of $\varepsilon=a_{1}-a_{0}$ :

$$
\begin{aligned}
& x=\frac{(1-\mu) \varepsilon+\sqrt{(1-\mu)^{2} \varepsilon^{2}+\mu^{2}\left(1-\varepsilon^{2}\right)}}{\mu(1-\varepsilon)} \\
& \alpha=\frac{\mu\left(x^{2}-1\right)\left(1-\varepsilon^{2}\right)}{4 \varepsilon l x} \\
& \beta=\frac{1-\varepsilon^{2}}{4 \varepsilon} \ln (x)
\end{aligned}
$$

with $0 \leq \varepsilon \leq 1$.

For $\varepsilon \rightarrow 0\left(a_{1} \simeq a_{0}, r \rightarrow 0\right)$ we have $T=\beta^{-1}=4 \mu+O\left(\varepsilon^{2}\right)$ and $\alpha=1 / 2 l$. This relationship confirms the intuitive corrispondence between temperature and mutation rate, at least for moderate differences in the tRNA abundances. For $\mu \rightarrow 0$, we get $j_{\max }=l$, corresponding to a distribution dominated by the fitter strains. Increasing the mutation rate $\mu, j_{\max }$ decreases; extrapolating to infinite value of $\mu$ (beyond the validity of our model), we get $j_{\max }=l / 2$, and the influence of selection is vanished by mutations. 
For $\varepsilon \rightarrow 1\left(a_{1} \gg a_{00}, r \rightarrow-\infty\right), T \rightarrow \infty$ and $\alpha=(1-\mu) / l$. In this limit only the fittest group $j=j_{\max }=l$ survives. In this case all mutations are deleterious, and their effect is to lower the growth rate $\alpha$ of the total mass of bacterial population.

At this stage of development, our model is too simplified to allow quantitative comparison with experimental data. However, it can be used as an interpretative tool for the understanding of the mutation/selection dynamics.

\section{PERTINENT DNA SEQUENCES ANALYSIS AND DISCUSSION}

In order to estimate the degree of optimization between codons and tRNA species we have analyzed 1530 Escherichia coli coding regions.

The choice of analyzing mainly the $E$. coli coding sequences depends not only on the large amount of data in literature and the large number of genes that have been sequenced (about $35 \%$ of the genome, at this date) but on the fact that in $E$. coli genome the effect of a directional mutational pressure towards an increase in $\mathrm{G}+\mathrm{C}$ or $\mathrm{A}+\mathrm{T}$ content is very little (about $51 \% \mathrm{G}+\mathrm{C}$ content), as compared with other organisms like Micrococcus luteus ( $74 \%$ $\mathrm{G}+\mathrm{C}$ content) or Mycoplasma capricolum (25\% G+C content) (Sueoka, 1993) (Osawa \& Jukes, 1988b). This pressure, probably due to mutations in the DNA polymerase system, acts particularly in shrinking the codon and anticodon sets: in E. coli there are 75 tRNA genes, and 45 types of anticodons, while in Micrococcus luteus or Mycoplasma capricolum these numbers are much lower (29 and 33 tRNA genes, resp.). Besides, the large effects of the directional mutational pressure on genomes composition probably results in weakening other existing functional constraints.

For each gene we have considered the simple relationship (四) between codons at position $i,\left(c_{i}\right)$ and tRNA molecule abundances $\left(\rho_{i}\right)$. We have considered equal translational efficiency for all codons.

The effective tRNA species abundances at different duplication rates are not exactly known; little differences in the total tRNA abundances have been proved experimentally among different strains of $E$. coli (Jakubowski, 1984), but the relative abundances do not seem to vary largely even among different species of enterobacteria: the data by Ikemura show that there is a good correspondence between the relative abundances of the tRNA species between E. coli and Salmonella typhimurium. Thus we have approached the problem performing the calculations in two ways:

a) considering the data on the relative abundances of the tRNA species published by Ikemura $\left(\tau_{\mathrm{I}}\right)$ (Ikemura, 1981a) (Ikemura, 1981b) (Ikemura, 1985) and Jakubowski (Jakubowski,1984).

b) using the data on tRNA gene dosage published by Komine $\left(\tau_{\mathrm{K}}\right)$ (Komine et al., 1990).

Both these two implementations are affected by different approximations.

In case (a) the data determined by Ikemura are not exhaustive. We have approximated the abundances of the minor tRNAs at the value 0.1 with respect to the abundance of LeutRNA normalized at 1 . In case (b) we have considered the abundance of tRNA species to be just proportional to the number of tRNA genes; this assumption could be not completely true because different tRNA gene clusters are regulated by different promoters and because of differences in aminoacyl-tRNA synthetases abundances and catalytic activities. 
In Fig. 1 we report the average time spent per codon $\tau_{\text {I }}$ for 1530 coding sequences, both for the correct reading frame and for the +1 and +2 reading frames. The sequences are ordered according with the value of $\tau_{\mathrm{I}}$ of the correct reading frame.

In Fig. 2 we report $\tau_{\mathrm{K}}$ for the same sequences of Fig. 1.

The comparison of the two figures shows that there is a good agreement between the values of $\tau_{\mathrm{I}}$ and $\tau_{\mathrm{K}}$, although the data obtained using the distribution of tRNA abundances from Ikemura show a more marked separation between the correct reading frame and the +1 and +2 frames. This indicates that there is a good correspondence between tRNA abundances and number of copy of the related tRNA gene, i.e. the most abundant tRNAs are expressed by triplicated or quadruplicated genes while the less abundant tRNA species are expressed by genes present in a single copy.

In all the analyzed coding regions, the correct reading frame always presents the lower crossing times, although the differences are more marked for the highly expressed sets: the mean translation time of the correct reading frame for highly expressed coding regions (as ribosomal genes, see Fig. 1) is lower than those calculated for non highly expressed coding regions, while the value for the +1 and +2 frames are equal or sometimes greater.

We have also compared our results with the Sharp and Li's Codon Adaptation Index $(C A I)$ of each sequence. This index does not depend directly on the abundances of tRNA but it is a measure of the bias in codon usage (Sharp \& Li, 1987) defined as follows

$$
C A I=\left(\prod_{k=1}^{l} w_{k}\right)^{\frac{1}{l}} ; C A I \in(0,1)
$$

where the product of $w_{k}=w\left(i_{k}\right)$ is taken over the code under examination. The quantities $w\left(i_{k}\right)$ for a codon $i$ at position $k$ is given by the relative frequency of the codon $i$ with respect to the most used codon for the same aminoacyd in a set of highly expressed genes. Note that in principle is possible to sinthetize artificial sequences made only of major codons with a $C A I$ value greater than that of the most abundant proteins. This occours because this index neglect the influence of mutations. The same misunderstanding occours with our quantity $\tau$.

In Fig. 3 we report the $C A I$ values for the same sequences of Fig. 1. The agreement with both the $\tau_{\mathrm{I}}$ and $\tau_{\mathrm{K}}$ is rather good.

The genes that show a highly biased codon usage have high values of $C A I$ (and low values of $\tau_{\mathrm{I}}$ ) and belong to the translation and transcription machinery, as for instance genes coding for ribosomal proteins, initiation and elongation factors, heat shock or stringent response proteins, genes involved in the core of intermediate metabolism and genes coding for the most abundant membrane proteins.

Generally the genes whose products are proved to be required for maximal growth rate show high value of $C A I$. We can consider for instance the ${ }^{118} I F 2 \alpha$ and ${ }^{71} I F 2 \beta$ (Sacerdot et al., 1992) that operate in the initiation of the translation process and probably affect the production of ribosomal RNA, and the product of the nusA gene that is involved in the transcriptional process. These three genes are clustered in the same operon, probably for a co-regulation of translation and transcription. The apices indicate the position of the sequences in the figures. 
The DNA polymerases I and II are present in different concentrations; about 400 molecules of polymerase I per cell with respect to 17-100 molecules per cell of polymerase II (Adams et al., 1992). The $C A I$ values of the two genes $\left({ }^{334} \mathrm{pol} A\right.$ and $\left.{ }^{760} \mathrm{polB}\right)$ reflect the difference: $0.403\left(\tau_{\mathrm{I}}=2.29\right)$ versus $0.352\left(\tau_{\mathrm{I}}=2.89\right)$.

Since biosynthetic operons are quite constitutively expressed, except during starvation conditions, the genes coding for the repressor proteins show $C A I$ values lower than repressor genes in catabolic operons.

In some cases genes coding for functional aggregates constituted by structural proteins interacting in a precise stoichiometry ratio show values of $C A I$ or $\tau_{\text {I }}$ close to this ratio. This relation is true only for highly expressed genes; for example some ribosomal proteins, as ${ }^{1} L^{7}$ and ${ }^{11}$ L20, are present as a tetramer in each ribosome (Adams et al., 1992): the $C A I$ value of the correspondent genes are higher than those of other ribosomal genes; the same correlation has been found for ATP synthetases genes that code for a large complex constituted by many copies of different subunits: there is a good agreement between the $C A I$ values of the genes and the stoichiometry of the subunits in the complex. This correspondence almost vanishes for structural proteins coded by low expressed genes as those coding for fimbriae, pili and flagella.

Generally, the enzymes that channel a metabolic pathway do not present the same bias in codon usage, probably because natural selection acts primary on the homogenization of the catalytic activity of the metabolon, however there are also few interesting clues of the contrary.

The membrane lipids biosynthesis in E. coli involves the action of at least 25 genes; many of these are clustered in the $f a b$ operon. Although the regulation of this pathway is very complex and not yet completely elucidated, with regard to both the total fatty acid content and the phospholipids composition, there are some observations that indicate that the activities of few enzymes (acetyl-CoA:ACP transacetylase, acetyl CoA carboxylase) are rate limiting in vivo (Magnuson et al., 1993). We have found that the bias in codon usage of these genes is higher than that of the genes coding for the other enzymes of the pathway. Since growth rate depends also by membrane lipids production, the bias in the bottleneck of the pathway probably increases the rate of production, while its effect on the other genes is vanishingly small.

Other interesting examples regard the relatively high bias in codon usage in genes like ${ }^{24}$ spo T, ${ }^{180} \mathrm{htpr},{ }^{280}$ usp A, ${ }^{295}$ fis, ${ }^{130} \mathrm{csp} A$ and ${ }^{175}$ ack $A$.

Since spoT encodes for a ppGpp pyrophosphohydrolase, it takes into account for the degradation of ppGpp, the major inductor of the stringent response, that accumulates during starvation periods.

Nutritional shift-up experiments, in which large amount of nutrients are added to bacteria growing in a minimal medium, have revealed that there are immediate changes in the rate of increase of cell mass while the cell division rate continues for some time at pre-shift rate (Cooper, 1991).

Thus the competition among bacteria requires to exit quickly from the starvation periods and to restart growing when conditions have changed; in this sight the spoT gene $(C A I=$ $\left.0.59, \tau_{\mathrm{I}}=1.4\right)$ could be a very important target for natural selection. Probably for the same reason the $h t p r$ gene $\left(C A I=0.553, \tau_{\mathrm{I}}=1.95\right)$ has a relatively highly biased codon usage: the encoded protein coordinates the global response of the cell to heat shock events. In fact 
this gene codes for a sigma-32 subunit that substitutes the sigma-70 in the core of RNA polymerase making the new holoenzyme capable of activating the heat shock promoters. Henceforth other heat shock genes show a biased codon usage as for instance ${ }^{485} \mathrm{dnaJ}$ and ${ }^{462}$ groEL. The intracellular amount of the universal stress protein, coded by the gene usp $A$ $\left(C A I=0.52, \tau_{\mathrm{I}}=2.19\right)$, greatly increases in the cases of exhaustion of nutrients like carbon, nitrogen, phosphate, sulfate or aminoacid (Nystrom \& Neidhardt, 1992).

We hypothesize that codon usage reflects not only the effects of selection for high growth rate conditions but also selection for very fast, and perhaps short time lasting, response to equally rapid environment changes. A clue that agrees well with this hypothesis concerns the fis gene $\left(C A I=0.53, \tau_{\mathrm{I}}=2.21\right)$ whose product functions as a DNA binding protein, involved in recombination reactions and in rRNA transcription. It has been reported that when the availability of a rich medium allows the bacterial cells to exit from the stationary phase, the amount of the fis gene product increases from less than 100 copies to over 50000 copies before the first cell division. As the exponential growth goes on, the Fis levels decrease considerably (Ball et al., 1992). The gene $\operatorname{csp} A\left(C A I=0.808, \tau_{\mathrm{I}}=1.81\right)$ codes for the major cold shock gene (CS7.4) (Tanabe et al., 1992); when the temperature shifts from $37^{\circ} \mathrm{C}$ to $10{ }^{\circ} \mathrm{C}$ the level of the protein largely increases and becomes about $13 \%$ of the total proteins in the bacterial cell.

It is noteworthy that since acetyl phosphate seems to be a global regulator of signal transduction in $E$. coli, also the gene ackA $\left(C A I=0.66, \tau_{\mathrm{I}}=1.9\right)$ that codes for a protein that synthesizes acetyl phosphate from ATP and acetate presents a high value of $C A I$.

There are genes with little if any differences in values of $\tau_{\mathrm{I}}$ between the correct reading frame and the others. This is mostly true for genes in plasmids (see Fig. 1). Since plasmids and conjugative transposons play a key role in the interchange of drug resistance and virulence traits among bacteria, and generally in the mobilization of the genetic material, it has been proposed that the entire pool of genetic information could be accessible to all members of the bacterial community, considered as a single, heterogeneous organism. In this regard the emergence of a bias in codon usage for a gene carried by a plasmid is treated unfairly by natural selection if the rate of the genetic information interchange among conjugative bacteria of different species is sufficiently high. In fact because of the genetic flux, the plasmid could visit a wide range of hosts, thus it could assay different molecular environments and therefore the recombinant processes between homologous genes maintain sequence homogeneity. Furthermore, an increased expression of a gene could be attained also by increasing the number of copies of the plasmids .

The debate between neutralists and selectionists in evolutionary and population genetics addresses the question of how the superposition and the interactions between the different constraints acting on the coding regions allow the tremendous amount of molecular genetic variation that natural bacteria populations exhibit.

The comparison of the standard deviation of the $C A I$ index for thirteen sequences of Dglyceraldehyde-3-phosphate dehydrogenase gene $\left({ }^{33}\right.$ gap gene; average $C A I=0.830$, standard deviation 0.0025) from different strains of E. coli sequenced by Selander ( Selander et al., 1991) and ten sequences of the alkaline phosphatase gene $\left({ }^{843}\right.$ pho $A$ gene, $C A I=0.344$, standard deviation 0.0059) sequenced by Milkman (DuBose \& Hartl, 1991), shows that genes with higher bias in codon usage present lower levels of silent polymorphism.

This relation suggests that the more biased the codon usage of a gene among a bacteria 
population, the less neutral the synonymous mutation.

Another fundamental question regards if and in what measure the bias in codon usage participates in the control of gene expression. E. coli and bacteria in general are governed by robust and plastic regulative mechanisms and even if regulative circuitry are parsimonious in design, the present day gene networks have a large tool-kit of regulative mechanisms, both at the gene-protein and at the protein-protein interaction level.

Although the basic mechanisms act at the operon level, there are global mechanisms that allow the cell to respond to challenges, as hunger or stress situations, with the coordinate and concerted expression of a network of operons. These mechanisms rely mainly in a cascadelike activation of autogenously regulated stimulons or in the sigma subunit variability that makes the polymerases capable of recognizing different types of promoters.

Within the operon there are mechanisms that differentiate the expression at the single gene level, as for instance internal promoters, transcription termination signals, ribosome binding sites with different efficiency, mRNA degradation signals etc.

Although bias in codon usage has been proved to play a role in differentiating the expression levels of the genes within the operon, since these mechanisms act sequentially, the effect of codon bias on the translation process probably depends largely on the optimization of the other factors involved in the gene expression.

The amounts of the genes that present high values of $C A I$, for instance the ribosomal and major outer membrane genes whose products are strongly required with respect to other proteins for high duplication rates, are the effective fitness bottleneck of the bacterial duplication machinery; thus the growth maximization strategy allows a cell to duplicate faster because it lowers the time needed for translation.

The natural selection acts so much stronger in optimizing the translation of the very highly expressed genes, with respect the other genes, because further increases in the translation efficiency of these latter genes reflects smaller improvements in the fitness of the cell.

Considerations of this type have also been proposed and tested by Dykhuizen and collaborators for lac operon in E. coli (Dean et al., 1986); they have demonstrated that the contribution to the fitness of the proteins encoded by the lac operon is quite different, being the bottleneck of the lactose metabolic pathway the flux of lactose into the cell, governed by the concentration of the enzyme lac permease: thus the increase of catalytic activity of the other enzymes coded by the lac operon (for example beta-galactosidase) results in a very little increase of the global fitness of the pathway.

This could be a general principle in gene network architecture, i.e. the potentiality of increasing the global fitness of the pathway could not be equally distributed among the genes belonging to a gene network.

Being generally accepted that the weaker the selective constraint, the wider the random genetic drift (Li \& Graur, 1991), the more relaxed situation of genes whose product are not required in large amount, allows high genetic variability consisting mainly in synonymous substitutions. 


\section{CONCLUSIONS}

We have modeled some aspects of the molecular evolution of bacteria genomes by means of the coupling between codon distributions and tRNAs abundance and the competitive effects of mutation and selection. Our model is consistent with a thermodynamical interpretation of this process, and gives a matematical support to the observed codon usage distribution.

The evolution of a non synchronized population of bacteria is schematically drawn as alternated phases of exponential growth (feast) and selection (famine), due either to starvation or to external reasons. In bacteria, because of the limiting amounts of nutrients and the rapid fluctuation in their availability in natural environment, the periods of growth are sporadic. Indeed, during these sporadic periods, the number of bacterial cells tends to increase exponentially and the competition between genotypes could develop very hardly, because a light difference in the fitness could result in a large difference in number and in the affirmation of the fitter genotype.

Probably the two extreme conditions of feast and famine model large part of the fitness function of the bacterial genomes.

Bias in codon usage seems to be an adaptive response not only to feast periods but also to conditions of changing surroundings. In fact to survive famine periods would require sophisticated regulative mechanisms leading to a shrewd management of the resources as for instance the stringent response, while the selection for rapid oscillating periods make the cells maximize the sharp up-shift or down-shift production of some specific proteins. According to this hypothesis, bias in codon usage has revealed an interesting tool to investigate the fitness bottleneck in metabolic pathways or in gene networks like stringent response.

\section{Acknowledgments}

We thank Fiammetta Battaglia, Marcello Buiatti and Stefano Ruffo for helpful discussions. 


\section{REFERENCES}

Adams, L., Knowler, J. T., Leader, D. P. (1992). The biochemistry of the nucleic acids Chapman \& Hall, London.

Ageno, M (1992). La macchina batterica (Lombardo ed.) Roma.

Andersson, G.E. \& Kurland, C.G. (1990). Microbiol. Rev. 5, 198-210.

Ball, C.A., Osuna, R., Ferguson, K.C., Johnson, R. C. (1992). J. of Bact. 174, 8043-8056.

Berry, A.J., Ajioka, J.W., Kreitman, M. (1991). Genetics 129, 1111-1117.

Bibb, M.J., Findlay, P. R., Johnson, M.W. (1984). Gene 30, 156-166.

Blake, R.D., Samuel, T.H., Nicholson-Tuell, J. (1992). J. Mol. Evol. 34, 189-200.

Chen, G.T. \& Inouye, M. (1990). Nucleic Acids Res. 18, 1465-1473.

Cooper, S. (1991). Bacterial Growth and Division. Academic Press, San Diego, California. Cowe, E. \& Sharp, P.M.(1991). J. Mol. Evol. 33, 13-22.

Dean, A.M., Dykhuizen, D.E., Hartl, D.L. (1986). Genet. Res. 48, 1-8.

DuBose, R. \& Hartl, D.L. (1991). In: Evolution at the Molecular Level (Selander, R.K. et al ed.) Sunderland, Massachusetts.

Emilsson, V. \& Kurland, C.G. (1990). EMBO J. 13, 4359-4366.

Emilsson, V. \& Kurland, C.G. (1990). Microbiol. Rev. 54, 198-210.

Eyre-Walker, A. \& Bulmer, M. (1993). Nucleic Acids Res. 21, 4599-4603.

Goldman, E. (1982). J.Mol. Biol. 158, 619-636.

Gouy, M. \& Grantham, R. (1980). Febs Letters 115, 151-155.

Grosjean, H. \& Fiers, W. (1982). Gene 18, 199-209.

Grantham, R., Gautier, C., Gouy, M., Mercier, R., Pave, A. (1980) Nucleic Acids Res. 8, 49-62.

Hoekema, A., Kastelein, R.A., Vasser, M., De Boer H.A. (1987). Mol. Cell. Biol. 7, 2914-2924.

Huynen, M.A., Konings, D. A.M., Hogeweg, P. (1992). J.Mol. Evol. 34, 280-291.

Ikemura, T. (1981). J.Mol. Biol. 146, 1-21.

Ikemura, T. (1981). J.Mol. Biol. 151, 389-409.

Ikemura, T. (1985). Mol. Biol. and Evol. 2, 13-34.

Jakubowski, H., and Goldman, E. (1984). J. of Bact. 158, 769-776.

Jukes, T.H., Osawa, S., Muto, A., Lehman, N.(1987). Cold Spring Harbor Symposia on Quantitative Biology. LII, 769-776.

Jukes, T.H., Ozeki, H., Umesono, K. (1988). Proc. Natl. Acad. Sci. Usa 85, 1124-1128.

Kano, A., Andachi,Y., Ohama, T., Osawa, S. (1991). J. Mol. biol. 221, 387-401.

Kawasaki, K. (1972). In: Phase Transitions and Critical Phenomena (Domb, C. and Green, M.S. eds.), Academic Press, London.

Kinnaird, J. H., Burns, P.A., Fincham, J.R.S. (1991). J. Mol. Biol. 221, 733-736.

Komine, Y., Adaki, T., Inokuchi, H., Ozeki, H. (1990). J.Mol. Biol. 212, 579-598.

Landau, L.D. \& Lifshitz, E.M. (1958). In: Statistical Physics, Pergamon Press, Oxford, UK. Li, W.H. \& Graur, D. (1991). Fundamentals of Molecular Evolution Sinauer Associates inc. Publishers, Sunderland Massachussets. 
Liljenstrom, H. \& von Heijne, G. (1987). J. Theor. Biol. 124, 43-55.

Liljenstrom, H. \& Blomberg, C. (1987). J. Theor. Biol. 129, 41-56.

Magnuson, K., Jackowski, S., Rock, C.O., Cronan, J. E. (1993). Microb. Rev. 57, 522-540. Menninger, J.R. (1978). J. Biol. Chem 253, 6808-6813.

Morton, B.R. (1993). J Mol. Evol. 37, 273-280.

Neidhardt, F.C., Ingraham, J.L., Law, J. (1987). In: Escherichia Coli and Salmonella Typhimurium: Cellular and Molecular Biology. (Neidhardt, F.C.et al., ed.) American Society for Microbiology, Washington, DC.

Nystrom, T. \& Neidhardt, F. C. (1992). Mol. Microb. 21, 3186-3198.

Jukes, T.H., Osawa, S., Muto, A., Leiman, N. (1987). Cold Spring Harbor symposium on quantitative biology LII, 769-776.

Osawa, S. \& Jukes, T.H. (1988). TIG 7, 191-197.

Osawa, S., Ohama, T., Yamao, F., Muto, A., Jukes, T., Ozeki, H., Umesono, K. (1988).

Proc.Natl. Acad. Sci. Usa 85, 1125-1128.

Osawa, S. \& Jukes, T.H. (1989). J.Mol. Evol. 28, 271-278.

Ohama, T., Muto, A., Osawa, S. (1990). Nucleic Acids Res. 18, 1565-1569.

Sacerdot, C., Vachon, G., Laalami, S., Morel-Deville, F. , Cenatiempo, Y., GrunbergManago, M. (1992). J. Mol. Biol. 225, 67-80.

Nelson, K.N., Whittam, T.S., Selander, R.K. (1991). Proc. Natl. Acad. Sci. Usa 88, 6667-6671.

Sharp, P.M. \& Li, W. (1986). Nucleic Acids Res. 14, 7737-7749.

Sharp, P.M. \& Li, W. (1987). Nucleic Acids Res. 15, 1281-1295.

Sharp, P.M., Shields, D.C., Wolfe, K.H., Li, W. (1989). Science 258, 808-810.

Sorensen, M. A., Kurland, C. G., Pedersen, S. (1989). J.Mol. Biol. 207, 365-377.

Sorensen, M. A., Jense, K.F., Pedersen, S. (1990). In: Post-transcriptional control of gene expression. (McCarthy and Tuite ed.) NATO ASI Series.

Sorensen, M. A. \& Pedersen, S. (1991). J.Mol. Biol. 222, 265-280.

Sueoka, N. (1993). J Mol Evol 37, 137-153.

Tanabe, H., Goldstein, J., Yang, M., Inouye, M. (1992).J. of Bacter. 174, 3867-3873.

Thomas, L.K., Dix, D.B., Thompson, R.C. (1988). Proc. Natl. Acad. Sci. Usa 85, 42424246.

Varenne, S., Buc, J., Lloubes, R., Lazdunsky, C. (1984). J.Mol. Biol 180, 549-576.

Varenne, S., Buc, J., Lloubes, R., Lazdunsky, C. (1986). J.Theor.Biol. 120, 99-110.

Veaute, X. \& Fuchs, R. (1993). Science 261, 598-601.

Yamao, F. , Andachi, Y., Muto, A., Ikemura, T., Osawa, S. (1991). Nucleic Acids Res. 22, 6119-6122.

Wright, S. (1932). Proceedings of the Sixth International Congress of Genetics 1, 356-366.

Wolfe, K. H. (1991). J. Theor.Biol. 149, 441-451.

Zyskind, J.W., \& Smith, D.W. (1992). Cell 69, 5-8. 


\section{FIGURES}

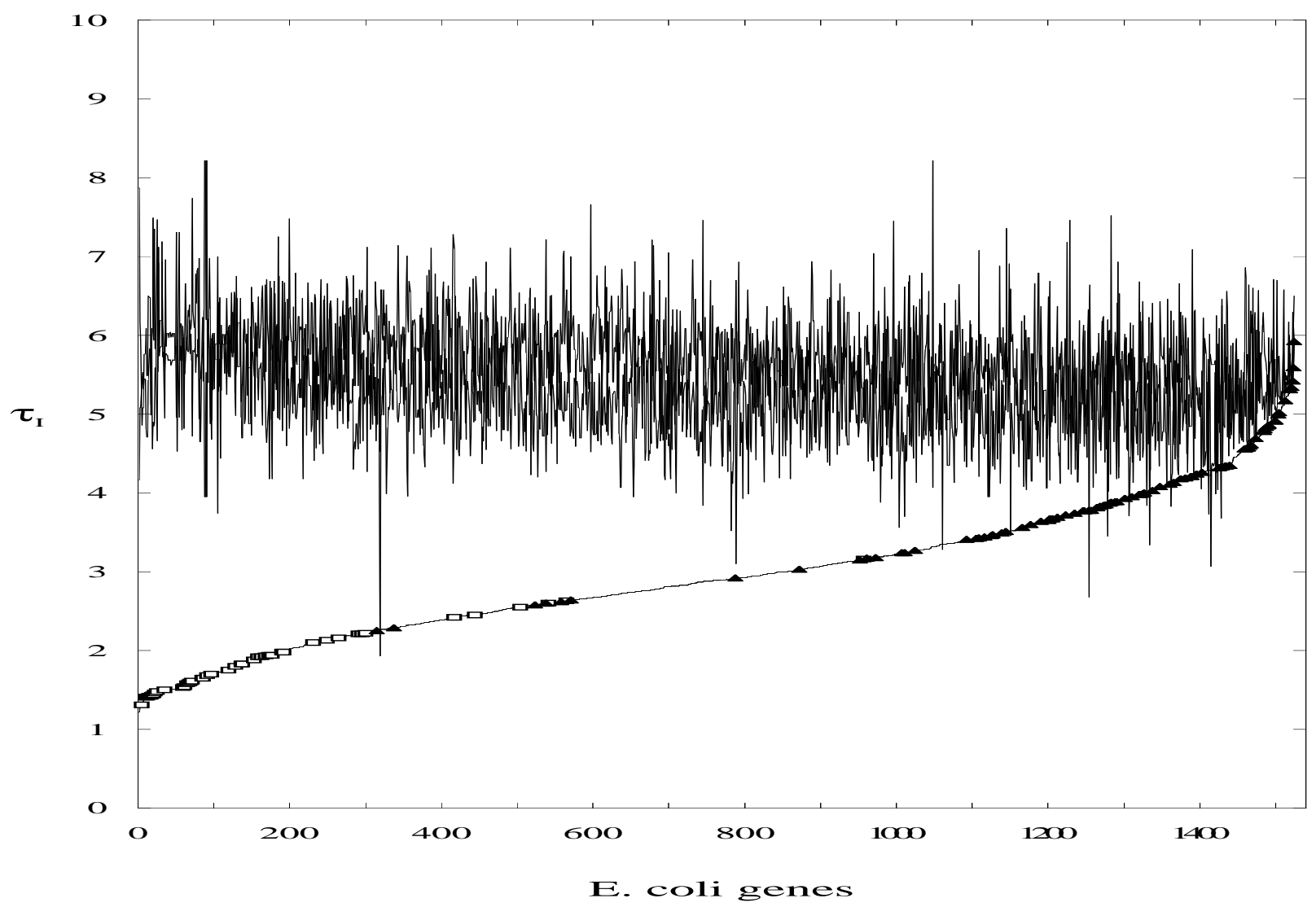

FIG. 1. Mean translation time $\tau_{\mathrm{I}}$ for 1530 Escherichia coli coding regions calculated using data on tRNA abundances published by Ikemura. The sequences are odered according to the values of $\tau_{\mathrm{I}}$. The lower line shows $\tau_{\mathrm{I}}$ for the correct reading frame. The upper lines show the value of $\tau_{\text {I }}$ for the +1 and +2 reading frames. The open squares correspond to ribosomal genes, the filled triangles correspond to genes carried by plasmids. 


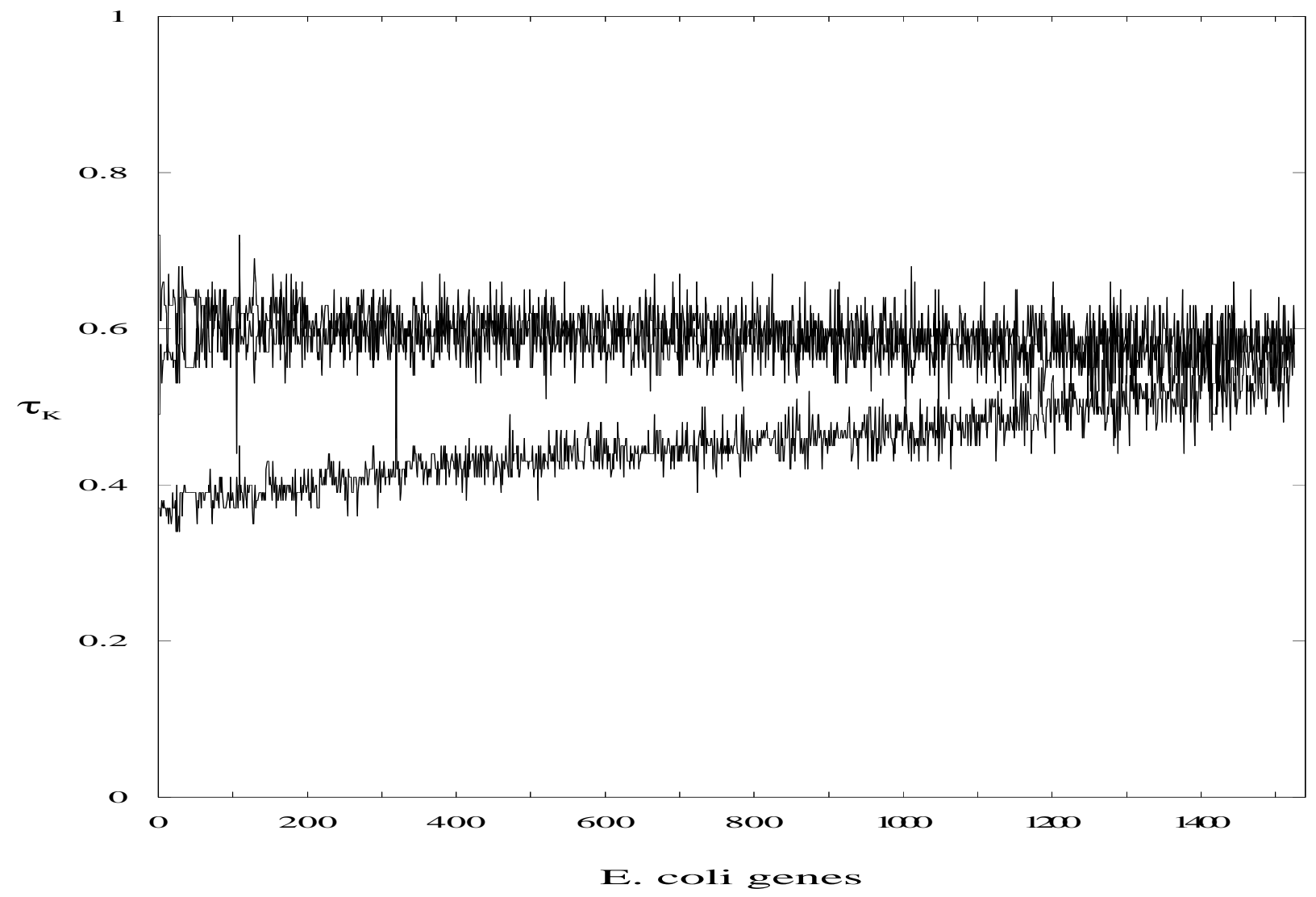

FIG. 2. Mean translation time $\tau_{\mathrm{K}}$ calculated using the data on tRNA gene dosage by Komine. The sequences are ordered as in Fig. 1. The lower line shows $\tau_{\mathrm{K}}$ for the correct reading frame. The upper lines show the value of $\tau_{\mathrm{K}}$ for the +1 and +2 reading frames. 


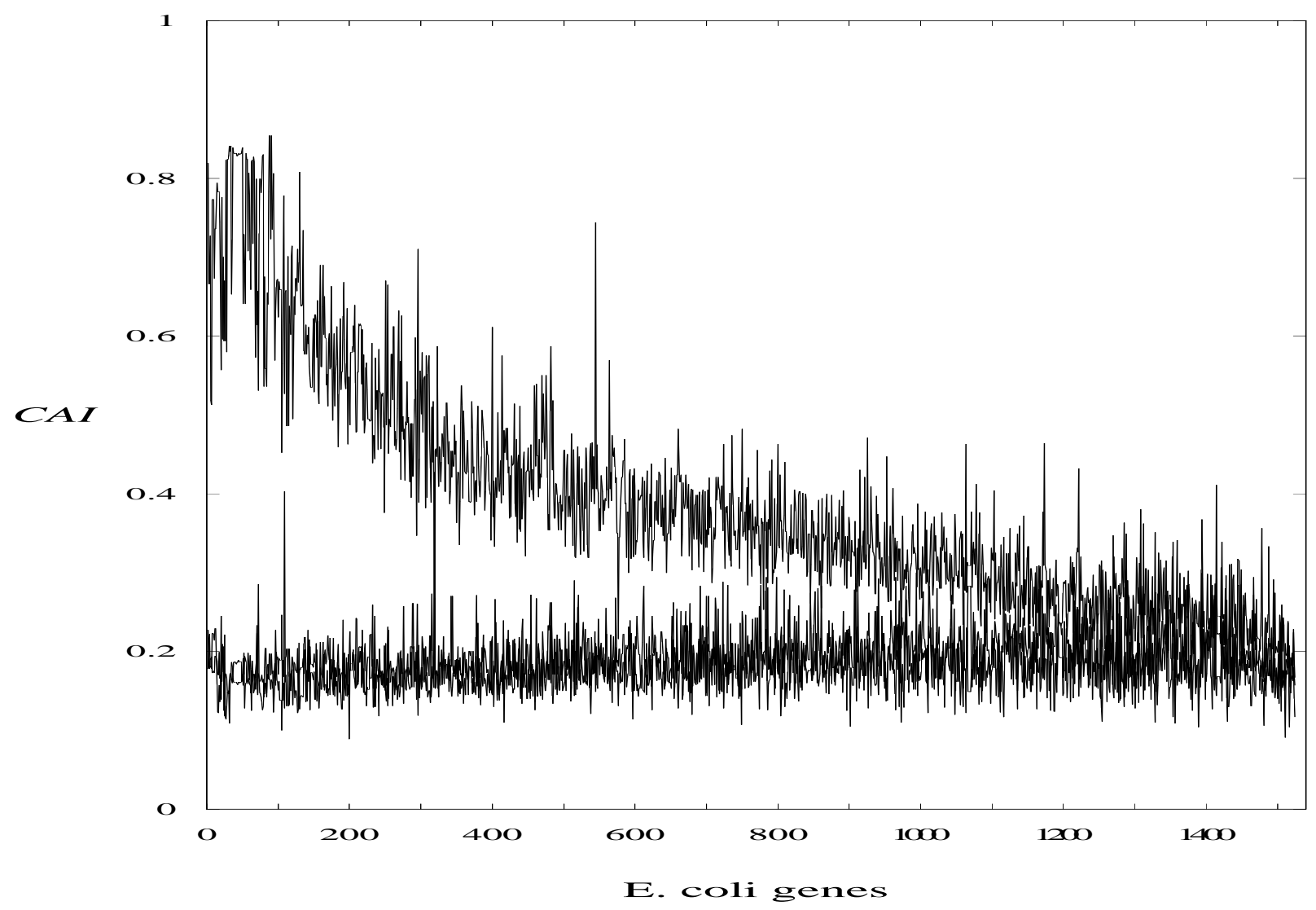

FIG. 3. $C A I$ values of the coding sequences ordered as in Fig. 1. The upper line shows $C A I$ values for the correct reading frame. The lower lines show $C A I$ values for the +1 and +2 reading frames. 